I N V E S T I G A C I Ó N

\title{
Una pedagogía narrativa para la formación del profesorado*
}

\section{A narrative pedagogy for Teacher Education}

José Contreras Domingo¹, Emma Quiles-Fernández², Adrià Paredes Santín³

Recibido: 11 de Junio de 2019 Aceptado: 15 de Julio 2019 Publicado: 31 de Julio de 2019

To cite this article: Contreras, J., Quiles, E. y Paredes, A. (2019). Una pedagogía narrativa para la formación del profesorado. Márgenes, Revista de Educación de la Universidad de Málaga, o (0), 58-75

DOI: http://dx.doi.org/10.24310/mgnmar.voio.6624

\section{${ }^{1}$ José Contreras Domingo 0000-0001-9242-756X \\ Departamento de Didáctica y Organización Educativa, Universidad de Barcelona jcontreras@ub.edu}

\section{${ }^{2}$ Emma Quiles-Fernández 0000-0002-9401-4387 \\ Departamento de Didáctica y Organización Educativa, Universidad de Barcelona emma.quiles@ub.edu}

\section{${ }^{3}$ Adrià Paredes Santín}

Departamento de Didáctica y Organización Educativa, Universidad de Barcelona adria.paredes@ub.edu

\begin{abstract}
RESUMEN
El presente artículo da cuenta del modo en que una pedagogía narrativa para la formación del profesorado puede ser entendida como una manera de acompañar a nuestras y nuestros estudiantes y a los modos desde los que desarrollan y configuran sus saberes docentes. Desarrollar un pensamiento y un saber narrativo no parte, necesariamente, de las vivencias y experiencias con las que llegan al aula (aunque estas han de poder estar presentes). Más bien se trata de cultivar un pensar narrativo que, como pensamiento relacional, posibilita una profundización pedagógica. Co-componer y pensar con las historias propias y ajenas nos evoca la pregunta de cómo nos preparamos para enseñar. Y de cómo nos preparamos para enseñar una pedagogía narrativa. Una preparación que tiene que ver con cómo cultivamos el saber narrativo en el aula universitaria y de qué manera la indagación narrativa es vivida como práctica pedagógica. En este sentido, el texto muestra no sólo diversas formas en las que la narrativa se hace presente, sino modos desde los que la formación puede ser un espacio para la indagación narrativa.
\end{abstract}

Palabras clave: formación del profesorado; indagación narrativa; pedagogía narrativa

\section{ABSTRACT}

This paper focuses on our experiences as teacher educators at the Faculty of Education at the University of Barcelona. It enhances how a narrative pedagogy might be lived as an educational practice to be alongside our students and their own ways to cultivate and shape their professional knowledge. To develop a narrative awareness and knowledge does not only require attending to the stories experienced by them before they are part of our university courses (although these expe-

\footnotetext{
* Este artículo es fruto del proyecto de investigación "Relaciones educativas y creación del currículum: entre la experiencia escolar y la formación inicial del profesorado. Indagaciones narrativas” (EDU2016-77576-P), financiado por la Agencia Estatal de Investigación (AEI) del Ministerio de Economía y Competitividad, y el Fondo Europeo de Desarrollo Regional (FEDER).
} 
riences must be present). We believe it is about cultivating a narrative thought that, as a relational one, it enables pedagogical deepening. Thinking with our own and others' stories also has allowed us to ponder about how we prepared ourselves to teach. During the process it became crucial to imagine how we three were preparing ourselves to teach and to share a narrative pedagogy with our students. This experience is related to how we cultivate a narrative knowledge and in what ways the narrative inquiry is lived as a pedagogical practice. In this sense, the paper shows a range of ways in which a narrative approach might be present in a classroom as well as a reflective note about teacher education as a space for narrative inquiry.

Keywords: teacher education; narrative inquirí; narrative pedagogy

\section{APEGADOS A LA EXPERIENCIA}

Promover una pedagogía para la formación del profesorado supone tener en cuenta la experiencia y traer al aula relatos de experiencias educativas. Son estas narraciones, propias y ajenas, las que nos permiten conectar con las dimensiones subjetivas de quienes las viven y con las circunstancias en que se producen. Una conexión que nos pone a pensar de manera singular: apegados a la experiencia. En este sentido, proponerse una pedagogía que coloca en el centro la narración de experiencias y su indagación significa asumir un riesgo, porque no siempre sabemos cómo va a ocurrir, o de dónde nacerá esta posibilidad, o incluso si dará de sí. Para esta ocasión queremos iniciar este texto revisitando una experiencia que narra el inicio de una posibilidad narrativa. Porque al pensar con esta historia de nuestra clase, podemos desplegar algunas cuestiones que han resultado significativas en la búsqueda de una pedagogía narrativa para la formación del profesorado.

Comienza la clase, tal y como venimos haciendo últimamente, con la lectura, hoy por parte de Sara1, del "cuaderno rodante"2 que venimos componiendo como grupoclase. Sara se coloca de pie, frente al resto del grupo, y empieza a leernos lo que ha escrito. La historia con la que nos invita a pensar es acerca de cómo se siente ella en la universidad. En su relato nos cuenta la extraña sensación que vive en las clases, en donde algo, que no sabe identificar, le impide intervenir, le frena a la hora de participar, de hablar en clase; cuando ella, nos dice, no era así en el instituto. Antes se vivía con desenvoltura, animada y decidida a expresar sus puntos de vista y a participar en los debates de las clases. Ahora, aunque las ideas le bullen en la cabeza, no se atreve a expresarlas. Tras la lectura, se inicia una breve y más bien tímida conversación en la que algunos expresan su forma de vivirse en la universidad. Entre unos y otros intentamos explorar algunas explicaciones acerca de qué es lo que puede haber cambiado entre los dos escenarios -instituto y universidad-, pero también en ellas y ellos, para que se hayan producido estos cambios. ¿Cuánto de todo esto se debe al modo en que funcionan estas instituciones? ¿Cuánto a la masificación de las clases en la universidad? ¿Cuánto a las formas de conocimiento académico que tienden a colocarnos en el lugar de la carencia, del no saber? ¿Cuánto al ambiente que se crea en una clase y

1. A fin de proteger la identidad de nuestras estudiantes, todos los nombres que aparecen a lo largo del texto son pseudónimos.

2. La noción y experiencia de "cuaderno rodante" la tomamos de Teresa Punta (2013) quien nos inspira en nuestro quehacer docente a través de las historias que compartió con nosotros en su visita a la Universidad de Barcelona, en noviembre de 2017. 
que quizás la propia clase podría cambiar? ¿Cuánto a las formas de comportarse en una época de nuestra vida en la que tendemos más a protegernos, a no exponernos? (Diario de clase de Pepe, abril de 2019).

El texto de Sara, como otros que se escribieron en el cuaderno, y las preguntas que con ellos nos hacíamos estaban en clara relación con algunas de las cuestiones que orientaron las clases de "Planificación, diseño y evaluación del aprendizaje y la actividad docente", del grado de Educación Primaria. Situar el sentido de la asignatura alrededor de considerar la vida del aula, y la manera en que esa vida puede ser creada y cuidada en la escuela, como un horizonte más amplio en el que pensar lo que el título de la asignatura nombra, nos llevó a atender cuidadosamente la vida que también nosotros estábamos cultivando en la universidad. Desde el inicio del curso, Pepe y Adrià se preguntaban, junto con el equipo de investigación del que Emma también forma parte, acerca de las diferentes formas en que la narrativa puede estar y hacerse presente en el aula universitaria. Una preocupación que movilizaba nuestras conversaciones desde hacía tiempo y que tenía que ver con hacer de las clases de formación inicial del profesorado un espacio de experiencia. Un espacio en el que también promover otras formas de saber que pudieran nacer de un pensamiento de la experiencia (Contreras, 2010; 2013; 2016). En particular, buscábamos promover un pensar de la experiencia en relación a sus historias de formación: aquellos relatos de sí desde los que pudieran pensarse en su transcurrir formativo, en relación a su pasado, a su presente y a su futuro. Relatos con los que tomar conciencia de su devenir y con los que formularse las preguntas que se les fueran abriendo al asumir el compromiso con su futuro como maestras y maestros.

El "cuaderno rodante" apareció para dar respuesta a la demanda que expresaron las y los estudiantes de compartir experiencias, al plantearles la pregunta sobre la vida de nuestra clase y lo que queríamos para ella. Tras escuchar su demanda, a Pepe se le ocurrió hacer circular un cuaderno para que quien quisiera pudiera recogerlo y traer algo para compartir. Un cuaderno desde el que, quizás, podía nacer la posibilidad de una experiencia. Desde los inicios, el cuaderno quedó abierto en cuanto a las posibles historias que las y los estudiantes podían compartir. Por ejemplo, sobre situaciones que habían vivido, o sobre escenas que observaban en distintos lugares y que les hacían pensar en cualquier faceta de la infancia y su educación. En definitiva, compartir experiencias y pensamientos con los cuales traer algo propio a la clase para convertirlo en asunto de conversación y reflexión pedagógica. Y durante un tiempo se convirtió en un espacio desde que el poder pensarse en clase y como clase.

Como expresaba en su colaboración otra estudiante, Clara: "En este cuaderno he visto la oportunidad de explicar lo que pienso, ya que, particularmente, cuando no me salen las palabras, las escribo." O según recogía Alicia en su reflexión final del curso: "[El cuaderno] me proporcionó un espacio seguro para expresar mis inseguridades como maestra y compartir mis pensamientos, así como para escuchar y reflexionar sobre las aportaciones de los demás." Estas palabras nos develan no solo cómo la clase les permitía pensarse en primera persona, sino en qué modo el cuaderno 
se había convertido en una parte más de ella, pues era una forma de ir haciendo clase, de irnos haciendo grupo que convivía, que compartía preguntas, experiencias, saberes y estudio. Los escritos se sucedieron como una narración conjunta que iba encadenando una conversación por escrito, a base de contar distintas historias personales entre su ser estudiantes y su irse pensando como docentes. Al final de cada lectura se iban apuntando nuevas personas que querían escribir para los próximos días, motivadas por lo que la historia de ese día les había dado a pensar. Se iba componiendo así una historia común y plural que fue mostrando la trama variada y compleja de la clase. Una trama que combinaba, como en la historia de Sara, un presente en relación a su pasado; o como en el de Félix, distintos presentes, distintos puntos de vista acerca de cómo puede vivirse en nuestra clase el estar callado en los momentos de conversación; o como en el de Clara, su presente (atreverse a romper su silencio) y su futuro (ganar confianza en ella misma mediante este ejercicio); o el relato de Cristian, acerca de los modos de ser y comportarse en distintos escenarios (escolar y no escolar) en diferentes momentos de su vida; o el de César, y la importancia de comprender la propia historia personal para entenderse y repensarse como maestro; o el escrito que nos leyó Alicia, en el que nos habló acerca del modo en que ella se va reinterpretando para crear su propio modo de relacionarse con las niñas y niños a quienes da clases de refuerzo; o el de Lidia, que a partir de una escena vivida con la madre de una niña a quien da clase, nos traía la pregunta sobre la formación que necesitan.

En este sentido, el cuaderno pasó a ser una forma de indagar en las dimensiones de lo vivido en los procesos de enseñanza en contextos escolares, haciendo consciente al grupo, junto con sus profesores, de diferentes dimensiones, capas, tramas y matices que estaban presentes también en la clase. Eran relatos en relación que daban pie a pensarse como clase y a vivirse como grupo, a la vez que iban abriendo las múltiples facetas que hay en juego en los procesos de formación, incluso más allá de lo que en el transcurso de la asignatura era posible profundizar. Adrià y Pepe procuraban resaltar dichas facetas y hacer consciente la relación entre el tiempo de narrarse y pensarse como estudiantes y las conexiones y transiciones en su irse haciendo maestras, maestros. Porque al narrarse estaban tomando consciencia de su historia y cómo la podían repensar. Porque en aquel momento experimentaban un triple ejercicio fundamental como experiencia formativa que tenía que ver con la escritura, la lectura y la conversación. Una escritura, lectura y conversación que se daban en primera persona, en conexión personal, y que se habían convertido en un ejercicio deseado que podían mantener vivo también en la escuela, como una forma de compartir experiencias y saberes, de pensar juntos, y de crear y cuidar la relación educativa.

Si bien la historia del cuaderno nos permitía apreciar el movimiento que se fue produciendo en el grupo y la atención a sus procesos de formación como algo que les involucraba en muchas facetas de sus vidas personales, sin embargo, sus narrativas de formación no se sostenían solo en este cuaderno. Las historias, los relatos y el pensar narrativo estaban presentes en nuestras clases de muchas otras formas: como relatos de sus experiencias escolares infantiles; a partir de la recuperación de momentos de su historia personal en relación a algún aspectos sobre el que es- 
tuviéramos trabajando en clase (por ejemplo, en relación a momentos en los que se habían sentido "vivos" a lo largo de su escolaridad; momentos en los que "estudiar" había tenido sentido para ellas/os; etc.); mediante narrativas orales (con visitas de otras maestras a nuestra clase) o escritos de maestras; con los relatos de experiencias escolares, fruto de nuestras investigaciones en escuelas (Contreras y Quiles-Fernández, 2017) o del trabajo como maestro de Adrià; a través de documentales audiovisuales. Y junto a estas variadas formas de traer a nuestro presente, a nuestras clases, historias en donde se nos manifestaba la vida escolar y las vidas de quienes la componían, procurábamos el desarrollo de conversaciones, así como ejercicios y tareas que se basaban en un pensar narrativo; esto es, un pensar con las historias, no sobre ellas; un pensar en el flujo de las historias, en el que las ideas que se van gestando muestran su conexión con los acontecimientos presentes en esas historias, a la vez que movilizan nuestro sentido de las cosas (Clandinin, 2013, pp. 29-30). Conversaciones, ejercicios y tareas que pretendían, por tanto, ir más allá de las narraciones para indagar en ellas y, al hacerlo, indagar en nuestro propio saber.

Paralelamente al proceso que vivían Pepe y Adrià en sus clases, Emma había incorporado la escritura de un diario de clase en la materia "Sistema Educativo y Organización Escolar" durante el semestre anterior, pero para ella la narrativa tomaba vida desde otra preocupación: hacer del aula un espacio propio en el que cada estudiante pudiera habitarlo desde sí. Y el diario, junto con la lectura de cuentos, la creación de narrativas visuales y los ejercicios epistolares, había posibilitado no sólo la experiencia y la reflexión, sino que la palabra hubiera movilizado a la escucha y a la conversación, haciendo que las historias, sus historias, pasaran a ser el centro de lo que acontecía en clase. Un centro en el que también se colocaban las tensiones. Al tratarse de una materia en la que el estudio de la legislación educativa emergía como el primer bloque temático del plan docente, resultaba importante el diálogo entre lo que institucionalmente movilizaba la legislación y sus propias experiencias escolares. Precisamente, ese vínculo entre lo propio y lo externo (los contenidos de legislación) generó un conjunto de tensiones institucionales en el grupo. Pero también eso fue lo que llevó a Emma y a sus estudiantes a buscar otros modos de ser y de estar en la clase. Modos que tuvieran que ver con quiénes eran y en quiénes estaban llegando a ser como futuras maestras (Bateson, 2011).

Aunque los tres impartíamos materias diferentes, coincidíamos en la necesidad y el deseo de recuperar una pedagogía narrativa para mostrarles (y mostrarnos) la transición que llevaba a nuestras y nuestros estudiantes de lo que vivían en la universidad, a lo que podían pensar ya también como docentes, vinculado a lo que podrían llegar a indagar, cuidar, preguntarse y desear para sus clases. Pero también para abrirles la posibilidad de poder vivir su experiencia de formación más allá de la adquisición de conocimientos teóricos y recursos prácticos. "Un más allá que no es en vez de" (Punta, 2013, p.14). Porque nuestra pregunta no es solo acerca de las diferentes formas en que la narrativa puede estar presente en la formación del profesorado, sino más bien acerca de cómo puede ser la formación un espacio para la indagación narrativa: para favorecer un pensar narrativo, en conexión con las historias, que promueva nuevas formas de relacionarse con la experiencia, para abrir así 


\section{N V E S T I G A C I Ó N}

nuevas preguntas, nuevos sentidos, nuevas posibilidades a nuestras vidas presentes y futuras.

\section{PREPARARNOS PARA ENSEÑAR}

\section{Dewey (1969) nos recuerda que}

vivimos siempre en el tiempo que vivimos y no en algún otro tiempo, y sólo extrayendo en cada tiempo presente el sentido pleno de cada experiencia nos preparamos para hacer la misma cosa en el futuro. Esta es la única preparación que a la larga cuenta para todo (pp. 58-59).

Pensar las clases que vivíamos con nuestros grupos y el modo en que las y los estudiantes participaban en ellas traía consigo un sentido particular de la enseñanza que, como nos recuerda Dewey, tiene que ver con un tiempo presente. Y es desde la intensidad de dicho presente lo que nos permite imaginarlo e imaginarnos en el futuro. La clase, en tanto que espacio generador de conversaciones, pensamiento, preguntas y saberes, no sólo preparaba a quienes iban a enseñar posteriormente, sino a quienes ocupábamos el lugar de maestros en el presente. De hecho, que en nuestras clases esto se convierta en motivo de reflexión, no es fruto de la casualidad, pues en el núcleo de nuestra forma de entender la formación se encuentra el deseo y la necesidad de abrir las preguntas fundamentales de la educación no solo como preguntas para un futuro ejercicio profesional (un tiempo que se compone más de imaginaciones que de certezas), sino como preguntas también acerca de nuestro presente (lo que vivimos y estudiamos en nuestras clases) y de nuestro pasado (de qué historias educativas estamos hechos y cómo siguen vivas en nosotros, Okri, 2014). Cuestiones centrales de la educación y la enseñanza, como la relación educativa, la vida compartida de un grupo y cómo vivir juntos -una pregunta que es educativa y es política-, las vidas singulares que cada cual va componiendo, y qué tipo de experiencias nos acercan el mundo y nos permiten comprenderlo, eran todas ellas cuestiones para nosotros en nuestras clases y no solo preguntas para ellas y ellos para un futuro abstracto, cuando sean docentes. En el fondo se trataba de poner en juego un posible modo de enseñar y aprender que no se disociara de nuestras propias vidas. Vivian Paley (2006, p.28) apunta que "nadie enseña en abstracto", porque el enseñar y el aprender no existen por sí solos, sino que somos personas las que enseñamos y personas las que aprendemos, con nuestras vidas, con nuestras historias, con nuestros deseos y con nuestras imaginaciones (Greene, 2000).

Precisamente por eso, en los cursos que cada uno de nosotros impartía en el grado de Maestro/a de Educación Primaria, se nos hacía presente la necesidad de un modo de pensar y de una experiencia de formación que nos involucrara aquí y ahora, que nos incluyera, que no nos disociara ni nos desconectara (Rodgers y Raider-Roth, 2006). Comprendiendo la complejidad y las narrativas institucionales, sociales, políticas y culturales en las que vivimos, las cuales muchas veces han conformado nuestras prácticas, nuestro pensamiento y nuestro lenguaje de un modo reduccionista. Comprendiendo también que, en muchas ocasiones, nuestras historias nos mues- 
tran grietas y aperturas para existir, pues las historias "hacen explícito lo implícito, visible lo escondido, dan forma a lo informe y hacen más claro lo confuso" (Chou, Tu y Huang, 2013, p. 60). Y esta era la búsqueda que se daba (y continúa dándose, de un modo u otro) en nuestras clases: hacer de la experiencia de formación un camino para indagar en esos escenarios, para reconocer las tensiones que nos generan, y para buscar algunas rendijas con las que ir afectando y remodelando algunas facetas de esos escenarios y de nuestra vida en ellos.

Nuestra experiencia docente nos muestra que los relatos de experiencia permiten, entre otras cosas, rescatar aquellos momentos en los que se dieron esas aperturas para existir. Además, el hecho en sí de narrarnos, o de poder participar de algún modo en un proceso narrativo, es una apertura para existir. Por ello, cuidar los procesos narrativos puede ser un camino para extraer, como plantea Dewey, "el sentido pleno de cada experiencia". No solo de nuestras experiencias, sino también de aquellas otras que nos llegan por medio de historias en las que se expresa la trama de acontecimientos, subjetividades y contextos en la que se vive y se transmite la complejidad vital de una experiencia. Historias en las que, como participantes y receptores, completamos su significado (Ricoeur, 1995). Y en las que, si seguimos profundizando, podemos abrir nuevos significados y sentidos. Tomar la fuerza de las experiencias, propias y ajenas, y profundizar en ellas para preguntarnos por el sentido y las posibilidades de la educación y de la enseñanza es un modo de prepararnos para seguir haciéndolo a lo largo de nuestra vida. Y puede ser un modo especial de prepararnos como maestras y maestros: como quien vive aperturas para existir y quien también las cuida en sus estudiantes; como quien reconoce su propia vida y la de sus estudiantes; como quien reconoce las tensiones en las que se mueve el oficio docente; como quien profundiza en las experiencias para preguntarse por las grietas que pueden abrirse en nuestro pensamiento, en nuestro lenguaje, en nuestras prácticas, en nuestras instituciones, para ampliar, junto a nuestros estudiantes los registros de nuestra existencia.

\section{CULTIVAR EL SABER NARRATIVO EN EL AULA UNIVERSITARIA}

A través de las narrativas compuestas por nuestras y nuestros estudiantes y compartidas en la clase, descubrimos que vivir y cuidar el sentido narrativo en el proceso de formación, nos ha ayudado también a captar y comprender el potencial de la narrativa como forma de convocar un saber educativo sensible. Y es que al acompañarlos en el acto de recontar y profundizar en sus experiencias, percibimos tramas de vida que se ponen en juego al escuchar historias y experiencias ajenas. Esto nos ayuda también a imaginar una pedagogía narrativa que toma dicho potencial para orientar y ofrecer un nuevo sentido a la formación del profesorado.

En cuanto que expresión de la experiencia, una narración nos trae a la presencia, de forma tentativa, la trama compleja, existencial y dinámica de acontecimientos y subjetividades, de situaciones de la vida y de quienes las experimentan. Una historia nos evoca el vivir en sus sensaciones del existir, antes que en sus explicaciones 
e interpretaciones de esos acontecimientos, de esas subjetividades (Ricoeur, 1995). De hecho, una historia nos puede poner frente a una situación que nos desconcierta, y no necesariamente frente a una comprensión de esta (Bruner, 2003). En este sentido, la propia experiencia, como las narrativas orales compartidas en clase, si nos afectan, si nos tocan, nos reclaman pensar. Un pensar que acompaña a la experiencia y que invita a profundizar en ella para preguntarse, para sorprenderse, para tomar consciencia de las diferentes capas de que puede estar compuesta (Contreras y Pérez de Lara, 2010). El saber narrativo es el que vuelve una y otra vez al relato, para seguir pensando con él, para excavar en nuevas capas, para hacerse más sensible a lo que en él se nos muestra y a la experiencia que evoca. El saber narrativo, al estar en conexión con los relatos que nos traen experiencias vividas, y que nos traen por tanto a nuestra presencia las vidas de sus protagonistas, es un saber que no se puede disociar de esas vidas, de las personas de las que esas historias nos hablan y de las resonancias que nos provocan (Conle, 1996). Es un saber sensible a la subjetividad y a la alteridad. Por eso, en nuestras clases necesitamos volver una y otra vez a las historias de nuestros y nuestras estudiantes y a las experiencias que con ellas podemos evocar. Eso nos permite conectar mejor con sus narrativas, así como buscar nuevas y más profundas versiones de estas. Según lo expresa Margaret Olson (1995, p. 122), "el conocimiento narrativo se construye y reconstruye conforme componemos relatos para explicarnos a nosotros mismos y a otros". Ciertamente, reconstruimos el saber narrativo con aquellas narraciones que nos cuentan, no sólo nuestras y nuestros estudiantes, sino con las historias que también nosotros compartimos, como docentes, en nuestras conversaciones. $Y$ es que las experiencias ajenas mueven nuestra subjetividad, evocan nuestras propias historias, así como amplían "el horizonte de nuestra existencia" (Ricoeur, 1995, p. 152), abriendo posibilidades imaginativas para nuestras propias experiencias. Es así como componemos nuevas versiones de las historias ajenas. En ellas aparecen nuevos pensamientos, nuevas preguntas, nuevas posibilidades. Y ya no son historias ajenas, sino historias que nos conciernen, que nos involucran, que nos 'tocan'. A lo mejor, se trata de historias en las que nos reconocemos, o en todo caso, historias con las que nos pensamos. Por eso, el saber narrativo, como propone Olson (1995), es contextualizado, encarnado, y se encuentra siempre en proceso de elaboración, en especial, si se dispone de los contextos de relación en los que estas historias pueden ser expresadas y pensadas. Lo que cuenta como saber narrativo no es la acumulación de conocimientos que obtenemos de la experiencia, sino el proceso de su continua elaboración. "Si reconocemos el conocimiento narrativo como la construcción y reconstrucción de la experiencia, el foco es entonces la experiencia en sí misma." (Olson, 1995, p. 123).

En los procesos de formación que nosotros acompañamos desde la universidad, las historias y relatos de experiencia, se prestan a un pensar encarnado, porque tratamos de poner en movimiento la implicación personal y subjetiva de nuestras y nuestros estudiantes (ya sea como autores o como receptores). Un saber narrativo que, frente al paradigmático (como saber descontextualizado y despersonalizado), guarda estrechas relaciones con el modo en que nos vivimos en el flujo de la vida y de sus acontecimientos (Bruner, 1988). Y que es el modo en que nos vivimos en 
la experiencia de nuestro oficio educativo. En este sentido, practicar y cultivar la narrativa y el saber narrativo se ha convertido, para nosotros, en un modo que nos ayuda a prepararnos como docentes y que nos mantiene en una relación sensible y cuidadosa con lo vivido.

Por lo que a nosotros respecta, en nuestras historias de formación y relatos de experiencia como docentes universitarios, en diversas ocasiones hemos afrontado el conflicto entre las formas de conocimiento que nos han conformado y las historias vividas que nos convocaban a otros saberes y a otros modos de saber que no siempre conseguían expresión, profundización y reconocimiento. Precisamente eso ha sido lo que nos ha colocado en la necesidad de preguntarnos por el modo en que la universidad instituye la formación del profesorado y en la manera en que nuestra historia como profesores está afectada por las configuraciones institucionales; afectando, a su vez, a nuestras formas de concebir el saber pedagógico (tanto nuestros saberes como docentes, como aquellos que enseñamos a nuestros estudiantes). Pero también nos hemos podido preguntar por las grietas y aperturas que hemos encontrado en nuestra historia para existir como docentes y académicos; y por las rendijas que tratamos de encontrar y abrir en una época atrapada en las formas estandarizadas y monocordes de expresión y comunicación del conocimiento y obsesionada por el rendimiento, las evidencias, las competencias (Biesta, 2017).

\section{LA INDAGACIÓN NARRATIVA COMO PRÁCTICA PEDAGÓGICA}

Revisando ahora los cuadernos que invitamos a escribir a nuestras y nuestros estudiantes, sus narrativas escolares y los cuentos y textos narrativos co-compuestos en nuestras clases, se nos hace presente el modo en que la indagación narrativa nos invita a explorar y a profundizar en estas historias propias y ajenas como forma de cultivar un modo de saber. El trabajo desarrollado por Jean Clandinin y Michael Connelly (2000; Clandinin, 2013), centrado en el estudio de la experiencia educativa, parte de la convicción de que la experiencia (y la vida en general) se compone narrativamente. En este sentido, no solo vivimos experiencias que traducimos en relatos, sino que nos vivimos desde estos relatos que nos contamos; o desde aquellos relatos que nos han contado y desde las narrativas (sociales, culturales, institucionales) en los que están insertos y desde los lenguajes en los que se expresan. Por este motivo, la indagación narrativa, como modo de exploración de la experiencia y de los relatos en que se expresa, no se limita a contar historias. Por el contrario, su propósito es indagar en ellas, pensando narrativamente con la experiencia y con su relato, para aprender algo nuevo a través de la experiencia. No solo "de" la experiencia, sino "a través" de ella. Porque la experiencia es educativa no cuando confirma nuestros presupuestos, sino cuando nos trae algo nuevo no previsto. Y las historias son educativas cuando nos abren cuestiones y nuevas posibilidades para entender la experiencia, o para preguntarnos por aspectos del mundo y de la vida que no habíamos contemplado (Olson, 1995).

Además, en la indagación narrativa y en el cultivo de un modo narrativo de saber, es la exploración conjunta la que crea una comunidad educativa, en la que "todos so- 
mos profesores/estudiantes/aprendices al compartir nuestro conocimiento y nuestras preguntas sobre la enseñanza y el aprendizaje" (Olson, 1995, pp. 129-130).

Proponerse una pedagogía narrativa para la formación del profesorado significa acoger el proceso mediante el que las y los estudiantes van componiendo y reconfigurando su saber, a partir de sus historias. Porque el saber que nos sostiene como docentes emana de nuestro saber personal, de aquellas experiencias e historias en las que hemos ido configurando y sosteniendo el sentido de nuestra vida y de nuestro quehacer docente. Según lo expresa Jean Clandinin $(1992 ; 1993)$, damos sentido a nuestras prácticas de enseñanza a partir de nuestro saber de la experiencia, aquel saber encarnado, que procede de nuestras historias personales y que se activa en nuestras prácticas escolares y en nuestras vidas. La formación del profesorado es una parte de nuestro proceso de componer nuestras vidas como docentes. Formarse como docente es indagar en las historias que tenemos elaboradas acerca de la enseñanza. Viviendo otras historias y la posibilidad de contarnos otras historias, vamos reconfigurando nuestro saber.

Desde este punto de vista, una pedagogía narrativa para la formación del profesorado puede entenderse como una especial atención al desarrollo en nuestros y nuestras estudiantes de su saber docente, entendido como un saber encarnado, en continua configuración, teniendo en cuenta las vivencias con las que llegan, las experiencias que puedan darse durante la formación y las que imaginan y desean para su vida como enseñantes. Un proceso continuo, para que en su posterior vida profesional puedan vivirse siempre así, en este proceso de ir reinterpretando sus experiencias para ir generando nuevas comprensiones y nuevas disposiciones para su vida escolar con las niñas y niños con quienes la compartirán. La experiencia de formación podría entenderse como una permanente composición de sus historias de formación; "una continua reescritura de las vidas de los estudiantes, y no como una preparación para algo desconectado de lo que viene de antes y un entrenamiento para lo que vendrá después" (Clandinin, 1993, p. 11).

Pero esta reescritura requiere de aquellas condiciones que la desencadenan y la enriquecen. Pues, como argumentan Schaefer, Downey y Clandinin (2014, p. 208), la cuestión no es valorar o celebrar todos los saberes que traen consigo las futuras maestras y maestros. "Se trata más bien de comenzar a hacer visibles las complejidades de su saber y abrirlo a indagaciones que nos reclamarán, a ellos y a nosotros, qué sabemos, cómo sabemos y qué necesitamos sostener para vivir nuestras vidas como docentes y como personas". Si se concibe la formación como un continuo contar y recontar las historias con las que se van haciendo y pensando como maestras y maestros, necesitamos disponer de una comunidad que escuche y que cuide un clima de confianza, para poder generar un espacio conversacional en el que despertar interrogantes y disponerse a la exigencia intelectual. Y necesitamos también que esta conversación se enriquezca con múltiples voces; no solo las voces personales de la comunidad, sino también aquellas otras voces invitadas mediante lecturas, perspectivas teóricas y trabajos de investigación, imágenes y documentales, cuentos y otras narrativas, escritas o visuales; junto con escenas de vida y las palabras de 
maestras y maestros, y también las de niñas y niños. Es de todo este conjunto de ingredientes y voces participantes en las conversaciones de la comunidad del aula universitaria de donde puede venir la apertura a nuevas lecturas de la experiencia vivida. De donde puede venir también la percepción y la comprensión sensible a la vida escolar y a las vidas de quienes forman parte de ellas. Y como puede ampliarse el abanico de sentidos y posibilidades de lo educativo y el de su imaginación como docentes.

Mientras nuestras y nuestros estudiantes participan de esta dinámica de componer y recomponer sus relatos de experiencia y sus historias de formación, nosotros, como profesores universitarios, también estamos implicados "en un narrar y volver a narrar reflexivo" (Clandinin, 1992). Atender a sus narraciones nos lleva a tener que aprender a escuchar sus historias, a participar en ellas, acompañándolos y orientándoles para que realicen nuevas lecturas de estas, y a compartir nuestras historias para que puedan ampliar las suyas. Este "narrar y volver a narrar reflexivo" de nuestros procesos de formación nos puede ayudar a reconocer nuestra historia de formación, normalmente anclada en la tradición académica del conocimiento paradigmático (Bruner, 1988), mientras exploramos en nuestras clases la apertura al reconocimiento y a la profundización de los saberes narrativos. Nuestra indagación narrativa como docentes es una forma de recuperar nuestras historias de experiencias pasadas y presentes para poder comprender mejor nuestras transiciones, nuestras dificultades personales y los resquicios que exploramos en nuestro escenario institucional. En este proceso de indagación sobre nuestra experiencia imaginamos nuevas historias que nos abren posibilidades para nuestro quehacer docente.

En el sentido en que aquí lo estamos planteando, no es una propuesta de actuación ni un plan de formación. Es más bien una forma de mirar al sentido de la formación del profesorado que cuente con la propia experiencia de estudiantes y profesores, para poder así componer sus propios procesos de formación, reconociendo su sentido personal, experiencial y narrativo. La pedagogía narrativa no es tampoco una formulación especulativa. Por el contrario, es un modo de vivir y practicar los procesos de formación de una manera sensible a lo que realmente se vive en su transcurso, y como una forma de ir componiendo, estudiantes y profesorado, un saber en el que sostenerse; como estudiantes en sus procesos de formación y en su futuro docente; y como profesoras y profesores universitarios en nuestro continuo proceso de formación mientras acompañamos a estudiantes que quieren ser enseñantes.

\section{TRANSITAR LAS TENSIONES PARA HACERLAS FRUCTIFICAR}

Promover una pedagogía narrativa no es una tarea sencilla, ya que normalmente resulta disonante con respecto a las formas de saber predominantes en el ámbito académico y escolar. Tanto nuestros y nuestras estudiantes como nosotros nos hemos formado en una tradición del saber que valora y legitima los conocimientos abstractos y las explicaciones universales; que entiende el conocimiento como impersonal (aún se sigue insistiendo en muchas publicaciones académicas en la utilización de un lenguaje impersonal) y descontextualizado. Formas de conocimiento 
que se apoyan en lo que Bruner (1988) llama "pensamiento paradigmático". Un pensamiento basado en la lógica argumentativa, que construye sistemas conceptuales para proponer modelos idealizados de descripción o de explicación de la realidad. Estas formas de conocimiento aspiran a convencer de su verdad; a diferencia de las formas narrativas de saber, que lo que procuran es mostrar "su semejanza con la vida" (Bruner, 1988, p. 23). Unas y otras formas de pensamiento y de saber se relacionan con la realidad de forma diferente. Pero también conducen a formas de imaginación diferenciada: mientras que el paradigmático promueve hipótesis para verificar, el narrativo promueve la imaginación sobre la subjetividad de las personas y el sentido dramático de la vida y de la condición humana. Y mientras que el paradigmático, como forma de saber, nos reclama una distancia personal, el narrativo requiere la implicación subjetiva (Nussbaum, 1997).

Esta perspectiva narrativa que venimos incluyendo en nuestras clases desde hace ya algunos años, nos mostró ya desde nuestros primeros tanteos que en las historias de nuestras y nuestros estudiantes se evidencia esa disonancia entre las formas de saber bajo las que han construido su historia de relación con el conocimiento escolar y las, en ocasiones, difíciles transiciones que tienen que hacer para entender y habitar una pedagogía narrativa. Esto nos llevó a pensar que, acostumbrados a "un lenguaje codificado y desconectado de las experiencias significativas de sus vidas" (Clandinin, 1993a, p. 2), no siempre les resulta fácil pensar y escribir en conexión personal, contando con su experiencia, con lo que saben; no siempre encuentran sensato que de sus experiencias puedan nacer comprensiones y decisiones que les movilicen como maestras y maestros. 0 quizás con más precisión, no siempre entienden que esa exploración pueda ser una actividad adecuada para su formación universitaria; porque de ella esperan otra cosa, otro saber.

El relato instituido sobre la universidad está más asociado al conocimiento paradigmático, argumentativo, codificado y desconectado de nuestras vidas. Y nuestra "historia epistemológica" que se ha ido configurando en nuestra historia de formación, ha privilegiado este conocimiento y nos ha conformado según ese relato institucional con respecto a los saberes pedagógicos, y en especial, en lo que se refiere al saber didáctico. De la universidad parece "esperarse las soluciones a los problemas de la práctica" (Clandinin, 1993b, p. 179), y a la vez, desencantarse de ella por su incapacidad de cumplir esa promesa. La universidad parece aspirar a realizar dicha promesa, convencida de que el problema consiste en que aún no se ha encontrado el conocimiento que resolvería esos problemas, o bien que aún no se ha formado adecuadamente a las y los estudiantes en las competencias apropiadas (que muchas veces parecen reducirse a destrezas "entrenables") que les permitirá desenvolverse con éxito en las escuelas. Y la colocación inicial de las y los estudiantes suele ser esta de esperar aquel conocimiento que les proporcionará todos los recursos para desenvolverse con seguridad como docentes.

Sin embargo, la pedagogía narrativa no entiende la formación como la transmisión y adquisición de conocimientos y competencias-destrezas. No la entiende como la transmisión del conocimiento pensado "para" el profesorado, sino como el desarro- 
llo "del" saber docente (Clandinin y Connelly, 2004). Un saber docente en donde "la distinción entre lo personal y lo profesional se difumina" (Clandinin, 1993a, p. 2). Porque el saber en el que nos sostenemos como docentes no tiene suficiente con los conocimientos pedagógico-didácticos. Nos sostenemos como docentes con todo lo que nos va constituyendo y dando sentido. Porque somos personas que enseñamos. Hacerse profesor o profesora, maestra o maestro, es un modo de vivir, a partir de quien se es, de quien se va siendo. Y al hacerse docente, al irse haciendo docente, uno va componiendo una coherencia narrativa en la que se busca dar sentido a su vida (Schaefer, Downey y Clandinin, 2014). Precisamente porque "el oficio docente se hace con uno mismo" (Contreras, 2010, p. 64), no es suficiente con un conocimiento profesional que se disocie del sentido y de la composición de la propia vida. No es tan solo una mixtura entre aspectos personales y competencias profesionales. Es más bien cómo vamos componiendo nuestra vida, como vamos elaborando y reelaborando nuestra historia personal para ir integrando los compromisos del oficio docente que vamos captando, asumiendo y convirtiendo en decisiones y acciones.

Estos contrastes y disonancias entre las diferentes formas de concebir el conocimiento, los saberes que nos sostienen en el oficio docente y la formación del profesorado, son fuente de desconciertos y tensiones para nuestras y nuestros estudiantes, como lo son también para nosotros como formadores. Algunas de estas tensiones son reflejo de las dificultades con las que nos encontramos al proponer, en nuestras clases y en la universidad, una pedagogía narrativa. Y es que, a pesar de valorar la potencialidad de esta, sabemos que en la universidad continúan predominando otra lógica y otras demandas. Más aún, en una época de capitalismo cognitivo, dirigido a "hacer de la inteligencia (...) una fuerza productiva", sin saber dónde queda "la inteligencia como potencia reflexiva y autónoma" (Garcés, 2017, p. 62). En una época "de impaciencia", en la que se quiere una enseñanza consistente, segura y anticipable, dirigida a "la producción eficaz de resultados predecibles de enseñanza” (Biesta, 2017, pp. 19-22).

Por otro lado, nos hemos encontrado con otras tensiones que reflejan, más bien, las dificultades para aceptar y movernos con comodidad en una forma de ir sabiendo y aprendiendo que no nos sitúa en una relación de adquisición y posesión de conocimiento, sino en un proceso de transformación personal (Fromm, 1978). En diversas ocasiones, este hecho llevó a Emma a crear, en su clase, un espacio de conversación con las y los estudiantes. Un espacio desde el que cuidar y acompañar aquello que, como grupo, estaban transitando. Y es que conversar acerca de las tensiones y complejidades experimentadas formaba parte del modo en el que iban co-componiendo la narrativa del aula. El movimiento que Emma deseaba que sus estudiantes vivieran tiene que ver con lo que Pepe y Adrià les planteaban en el cuaderno rodante: que su formación la experimentaran como un proceso de indagación en el que los saberes se conectan y encarnan. Y en el desarrollo de una pedagogía narrativa, los tres coincidimos en que nos ha supuesto concebir los cursos como un proceso de indagación. Esto nos ha requerido, como indica Clandinin (1993b), abandonar la seguridad de las asignaturas preparadas antes de que empezara el semestre, con todos los contenidos establecidos, las lecturas seleccionadas, las tareas y pruebas de evaluación decididas... para aceptar la incertidumbre de un proceso abierto de indagación que 
ha ido naciendo de lo que se ha ido dando en cada uno de nuestros grupos de estudiantes, dando lugar a la continua construcción y reconstrucción del saber.

Esta tensión nos ha resultado todavía más aguda cuando las tendencias que se han impuesto en la universidad (justificadas por políticas de transparencia y de garantía por "el logro competencial") pretenden tener establecidos y controlados todos los procesos y resultados previsibles, funcionando como políticas de control y de autocontrol del profesorado. Repensar la narrativa institucional en la que habitamos y de la que formamos parte, nos permite explorar, descubrir y comprender mejor el modo en que esta condiciona y altera no sólo las narrativas de nuestras y nuestros estudiantes, sino también nuestras narrativas como docentes. Quizás este sea un proceso más difícil para nosotros que para nuestros estudiantes, pues lo que hemos podido descubrir hasta el momento es que, a nuestros y nuestras estudiantes, a pesar del desconcierto inicial y la inseguridad que les genera, reconocen con rapidez lo que les aporta cuando encuentran que pueden ser autoras (y no solo receptoras) de un saber. Y cuando exploran las relaciones entre lo que nuestras asignaturas les ofrecen y el modo en que pueden con ello revisar sus historias educativas y personales.

Sin embargo, en esta tensión entre el desarrollo de un saber docente (que incorpora lo personal y que se va gestando en un proceso de indagación) y la adquisición de conocimientos, se nos hace presente otra tensión: el lugar que ocupa en una pedagogía narrativa la teoría, el conocimiento disponible, los "saberes constituidos" (Cifali, 2005, p. 179). Ya hemos avanzado antes que la pedagogía narrativa supone una comunidad que cuida la conversación. Y en esta conversación hay múltiples voces, entre ellas, las de las aportaciones del conocimiento disponible. O quizás mejor, las aportaciones de los textos que nos traen ideas, experiencias, pensamientos, construcciones teóricas, datos empíricos, propuestas. Decimos textos, mejor que conocimientos disponibles, porque todas estas fuentes de conversación nos llegan como voces, esto es, como textos con autoría que exponen, argumentan, defienden, cuestionan, sugieren. Es esta autoría la que nos permite la conversación, la que nos permite prestar atención a lo que un texto, y alguien que lo sostiene, nos traen para pensar. Y la que arraiga la conversación, para que no se vaya a la disquisición desconectada de la realidad, sino que nos conduzca siempre a la relación con algo y con alguien que nos ayudan a pensar.

La cuestión, por tanto, no es el saber teórico versus el saber personal, sino cuáles son las formas de relación con la teoría que nos permiten pensarnos en el mundo, para poder reconstruir nuestra historia. Aunque también qué cualidades de la teoría son las que colaboran en este pensarnos en el mundo, recontarnos y reconstruirnos en él. Porque lo que cuenta como aportación teórica para este pensarse en el mundo, son aquellas teorías que no representan el mundo, sino que actúan como "herramientas para desplazarnos y para aprender a percibirlo de otra manera" (Garcés, 2018, p. 21). Cuando se vale de conceptos que "no capturan sentidos, sino que son llaves que abren caminos, los caminos de lo impensado" (ibíd.). Cuando propone palabras críticas que abren "una distancia entre nosotros y el mundo". (ibíd., p. 94), que nos permiten mirar al mundo y a nosotros mismos de otra manera. Porque lo que cuenta no es tanto las teorías como acumulación de certezas, sino llegar a 
participar de la misma actitud para relacionarnos con la realidad educativa y para pensar nuestro lugar en ella. "No se trata de apropiarse de las conclusiones de los saberes elaborados, sino de reconocer que los saberes pedagógicos están vivos, que su aprendizaje es inabarcable e inagotable desde la perspectiva de acumular conocimientos y gestionar situaciones con ellos" (Blanco, Molina y Arbiol, 2016, p. 236).

Hay una última tensión que quisiéramos mencionar, y que es necesario transitar junto con nuestros y nuestras estudiantes; tiene que ver con la necesidad, para quienes se están formando para ser maestras y maestros, de disponer de recursos y estrategias para la acción. Una pedagogía narrativa no significa negar esta necesidad, o no abordarla. Pero sí mirarla desde otro lugar. No desde la posesión de repertorios prácticos, como si fuera un arsenal disponible que tan solo requiere entrenamiento para su utilización. Sino desde su relación, de nuevo, con una voz y una experiencia que cuenta, en las complejidades de la vida (que es lo que una historia siempre nos trae a nuestra presencia), lo que supone hacerse cargo de situaciones de la vida escolar y llevarlas a cabo. Lo que buscamos desde una pedagogía narrativa, o una pedagogía de la experiencia, es acercarnos a relatos de experiencias pedagógicas que se prestan a ser indagados narrativamente; con el propósito de poder explorar en esas historias las huellas de las vidas personales y de la vida que se sucede en una historia, lo que tienen para decirnos. Y poder prestar atención a lo que una experiencia hace con nosotros: a lo que moviliza en nosotros, como disposición y posibilidad, para vivir nuestra propia experiencia. Para podernos situar más allá de los modelos, más allá de los pasos y procedimientos señalados, y poder estar más atentos a las vidas de las niñas y de los niños que respiran es esas historias, y a nuestras propias capacidades y responsabilidades, para dar una nueva vida a lo que con una experiencia nos llega.

\section{RESONANCIAS}

Iniciar este texto revisitando algunas de las historias vividas en nuestras clases de formación del profesorado, es el modo que nos parecía más adecuado para mostrar algunas de las cuestiones fundamentales que tienen que ver con la indagación narrativa en los procesos de formación. Pero que tienen que ver, sobre todo, con las vidas: con nuestras vidas presentes y en relación; o con las historias a partir de las cuales recuperamos facetas de nuestra vida con las que pensarnos y pensar aspectos esenciales de la educación; o con aquellas otras historias que nos traen a nuestra presencia escenas de vida y acontecimientos escolares; o con las vidas que nuestros estudiantes van imaginando y construyendo como docentes, en relación a las vidas de sus futuros alumnos. La narrativa, y su profundización, nos permite estar atentos y sensibles a todo ello y, a la vez, desarrollar un modo de saber que es personal y relacional. Un saber que nace de las experiencias, propias o ajenas, con sus múltiples dimensiones y matices, y a la vez, sin acabar de despegarse de ellas, se abre a nuevos sentidos y posibilidades en nuestra existencia como docentes.

Volver ahora a las narrativas co-compuestas junto a nuestras y nuestros estudiantes nos permite recordar que no podemos pensar en la enseñanza en las escuelas si no 
prestamos atención a que lo primero es la vida que allí se da: las vidas de quienes allí viven y conviven. Pero para reconocer la vida que se da en la escuela tenemos primero que poder nombrar y atender a la vida que vamos creando y recreando en nuestras clases, junto a nuestras y nuestros estudiantes. Y es que mientras que pensábamos la vida en las escuelas y les invitamos a observarla como una experiencia motora (pues nos pone en movimiento), lo que acontecía en nuestras clases ha pasado a ser una exploración compartida. En este sentido, pensar una pedagogía narrativa para la formación del profesorado responde a un modo de explorar aquello que pasa y nos pasa en la clase, más que como un plan específico de actuación.

En diversas conversaciones en el bar de la facultad, a los tres se nos hacía evidente que la mirada a la escuela y a la infancia necesitaba entrelazarse con la mirada de lo que vivíamos en clase. Y es ese entretejido el que nos interpelaba a todos, profesores y estudiantes. Porque ambas miradas nos colocaban en un recorrido en el que no podíamos asegurar lo que sucedería. Porque contar con las vidas de quienes conviven en el aula y contar con la vida que acontece responde también al ritmo de la vida y de las relaciones. Un ritmo al que sólo es posible atender si escuchamos en círculos, pues los relatos de experiencia y las historias compartidas "se mueven en círculos. Ellas no se mueven en línea recta" (Metzger, 2009). Precisamente es esa escucha atenta y sensible, circular, la que hemos tratado de cultivar en nuestras clases. Para Emma, atender a dicha circularidad tenía que ver con invitar a las y a los estudiantes a descubrir nuevas historias, o historias que estaban ya dentro de aquella narrativa compartida, $o$ historias que nos resuenan al escuchar lo que se comparte. Todo ello mediado por la pregunta acerca del sentido educativo (Quiles-Fernández, 2016). Además, para Pepe y Adrià, atender a dichas interpelaciones respondía a un modo de cultivar y habitar una manera de saber y una manera de vivir el oficio educativo. Y es en nuestras clases, cada una con una particularidad determinada, donde buscamos una pedagogía narrativa para la formación del profesorado que no responde ni a un uso instrumental ni tampoco a un empleo paradigmático. Más bien responde al deseo de que, al compartir y escuchar en nuestras clases diversas historias, las nuestras y las de otras personas, también nuestras y nuestros estudiantes, cuando estén en las escuelas, empiecen a escuchar las historias de las niñas y los niños y a crear con ellas y ellos nuevas historias.

\section{REFERENCIAS}

Bateson, M. C. (2011). Composing a further life: The age of active wisdom. New York: Knopf Doubleday Publishing Group.

Biesta, G. J. J. (2017). El bello riesgo de educar. Madrid: SM.

Blanco, N., Molina, M. D. y Arbiol, C. (2016). Explorar las tensiones para transitar el abismo: buscando el sentido del oficio docente. En Contreras, J. (Coord.). Tensiones fructíferas: explorando el saber pedagógico en la formación del profesorado. Una mirada desde la experiencia (223-256). Barcelona: Octaedro.

Bruner, J. (1988). Realidad mental y mundos posibles: los actos de la imaginación que dan sentido a la experiencia. Barcelona: Gedisa.

Bruner, J. (2003). La fábrica de historias: derecho, literatura, vida. Buenos Aires: Fondo de Cultura Económica. 
Chou, M-J., Tu, Y. C. y Huang, K-P. (2013). Confucionism and character education: a chinese view. Journal of Social Sciences, 9 (2), 59-66.

Cifali, Mireille (2005). Enfoque clínico, formación y escritura. En Paquay, L. et al. (coords). La formación profesional del maestro. Estrategias y competencias. (pp. 170-196). México: Fondo de Cultura Económica.

Clandinin, D. J. (1992). Narrative and story in teacher education. En Russell, T. y Munby, H. (eds). Teachers and teaching. From classroom to reflection (pp. 124-137). Londres: The Falmer Press.

Clandinin, D. J. (1993a). Teacher education as narrative inquiry. En Clandinin, D. J., Davies, A., Hogan, P. y Kennard, B. (eds). Learning to teach, teaching to learn (pp. 1-15). New York: Teachers College Press.

Clandinin, D. J. (1993b). Learning to collaborate at the university: finding our places with each other. En Clandinin, D. J., Davies, A., Hogan, P. y Kennard, B. (eds). Learning to teach, teaching to learn (pp. 177-186). New York: Teachers College Press.

Clandinin, D. J. (2013). Engaging in Narrative Inquiry. London: Routledge.

Clandinin, D. J. y Connelly, F. M. (2000). Narrative Inquiry. Experience and story in qualitative research. San Francisco: Jossey-Bass

Clandinin, D. J. y Connelly, F. M. (2004). Knowledge, narrative, and self-study. En Loughran, J., Hamilton, M., LaBoskey, V. y Russell, T. (eds). International handbook of self-study of teaching and teacher education practices (pp. 575-600). Boston: Kluwer Academic Publishing.

Clandinin, D. J., Davies, A., Hogan, P. y Kennard, B. (1993). Epilogue. En Clandinin, D. J., Davies, A., Hogan, P. y Kennard, B. (eds). Learning to teach, teaching to learn (pp. 218222). New York: Teachers College Press.

Conle, C. (1996). Resonance in preservice teacher inquiry. American Educational Research Journal, 33 (2), 297-325.

Contreras, J. (2010). Ser y saber en la formación didáctica del profesorado: una visión personal. Revista Interuniversitaria de Formación del Profesorado, 24 (2), 61-81.

Contreras, J. (2013). El saber de la experiencia en la formación inicial del profesorado. Revista Interuniversitaria de Formación del Profesorado, 27 (3), 125-136.

Contreras, J. (2016). (Coord.). Tensiones fructíferas: explorando el saber pedagógico en la formación del profesorado. Una mirada desde la experiencia. Barcelona: Octaedro.

Contreras, J. y Pérez de Lara, N. (2010). La experiencia y la investigación educativa. En Contreras, J. y Pérez de Lara, N. (Comps.) Investigar la experiencia educativa (pp. 21-86). Madrid: Morata

Contreras, J. y Quiles-Fernández, E. (2017). Vivir y profundizar experiencias de enseñanza desde una perspectiva narrativa. En Contreras, J. (Coord.). Enseñar tejiendo relaciones: una aproximación narrativa a los docentes y a sus clases de Educación Infantil y Primaria (pp. 19-33). Madrid: Morata.

Dewey, J. (1969). Experiencia y educación. Buenos Aires: Losada.

Fromm, E. (1978) ¿Tener o ser? México: Fondo de Cultura Económica.

Garcés, M. (2017). Nueva ilustración radical. Barcelona: Anagrama.

Garcés, M. (2018). Ciudad Princesa. Barcelona: Galaxia Gutenberg.

Greene, M. (2000). Releasing the Imagination: Essays on Education, the Arts, and Social Change. San Francisco: Jossey-Bass.

Metzger, M. (2009). Writing for your life. A guide and companion to the inner worlds. New York: Harper Collins.

Nussbaum, M. (1997). Justicia poética. Santiago de Chile: Andrés Bello. 
Okri, B. (2014). A way of being free. UK: Head of Zeus.

Olson, M. (1995). Conceptualizing Narrative Authority: Implications for Teacher Education. Teaching and Teacher Education, 11 (2), 119-135.

Paley, V. (2006). El niño que quería ser un helicóptero. El empleo de la narración de historias en el aula. Buenos Aires: Amorrortu.

Punta, T. (2013). Señales de vida. Buenos Aires: Lugar Editorial.

Quiles-Fernández, E. (2016). Cuidar la relación: el sentido de la experiencia educativa. Una investigación narrativa. Tesis doctoral. Universidad de Barcelona.

Ricoeur, P. (1995). Tiempo y narración. México: Siglo XXI.

Rodgers, C. R. y Raider-Roth, M. B. (2006). Presence in teaching. Teachers and Teaching: Theory and Practice, 12 (3), 265-287.

Schaefer, L. Downey, C. A. y Clandinin, D. J. (2014). New possibilities for reimagining teacher education. En Clandinin, D. J., Schaefer y L. Downey, C. A. (eds.) Narrative conceptions of knowledge: Towards understanding teacher attrition (pp. 199-208). Bingley (U. K.): Emerald. 